\title{
Inhibition of LRP5/6-mediated Wnt/ $\beta$-catenin signaling by Mesd attenuates hyperoxia-induced pulmonary hypertension in neonatal rats
}

\author{
Deepthi Alapati', Min Rong' ', Shaoyi Chen', Cuihong Lin², Yonghe Li ${ }^{2}$ and Shu Wu'
}

\begin{abstract}
BACKGROUND: Hyperoxia-induced neonatal lung injury is associated with activation of $\mathrm{Wnt} / \beta$-catenin signaling. Lowdensity lipoprotein receptor-related proteins 5 and 6 (LRP5/6) are Wnt coreceptors that bind to Wnt ligands and mediate canonical Wnt/ß-catenin signaling. We hypothesized that inhibition of LRP5/ 6 by their universal inhibitor, Mesd, would attenuate hyperoxia-induced lung injury.
\end{abstract}

METHODS: Newborn rat pups were randomly exposed to normoxia or hyperoxia at $90 \% \mathrm{FiO}_{2}$ and injected intraperitoneally with placebo or Mesd every other day for $14 \mathrm{~d}$. On day 15, phosphorylation of LRP5/6 (pLRP5/6), expression of Wnt/ $\beta$-catenin target genes, cyclin D1 and Wnt-induced signaling protein-1 (WISP-1), right-ventricular systolic pressure (RVSP), right-ventricular hypertrophy (RVH), pulmonary vascular remodeling, alveolarization, and vascularization were measured.

RESULTS: Hyperoxia exposure markedly induced pLRP5/6, cyclin D1, and WISP-1 expression in the lungs of placebo animals, but they were significantly attenuated by the administration of Mesd. Mesd also significantly attenuated hyperoxiainduced pulmonary hypertension (PH) and pulmonary vascular remodeling. However, there was no effect on alveolarization or vascularization after Mesd administration.

CONCLUSION: This study demonstrates that LRP5/6 mediates pulmonary vascular remodeling and $\mathrm{PH}$ in hyperoxia-induced neonatal lung injury, thereby suggesting a potential therapeutic target to alleviate PH in neonates with severe bronchopulmonary dysplasia.

B ronchopulmonary dysplasia (BPD) is the most common and serious chronic lung disease of premature infants. Severe BPD is often complicated by pulmonary hypertension $(\mathrm{PH})$ that significantly increases the mortality and morbidity of these infants $(1,2)$. Although hyperoxia, mechanical ventilation, and inflammation are known to disrupt normal sequence of lung development and lead to $\mathrm{BPD}$ and $\mathrm{PH}$, the underlying molecular mechanisms are poorly understood $(3,4)$, resulting in a lack of effective treatment for BPD and PH. Therefore, it is necessary to investigate pathogenic mechanisms in animal models and identify potential therapeutic targets.
Hyperoxia-induced lung injury in neonatal rodents is widely used as an experimental model for BPD. Chronic hyperoxia exposure induces inflammatory responses, disrupts normal lung development processes, and triggers a cascade of dysregulated reparative pathways that results in alveolar simplification, decreased pulmonary vascular development, excessive pulmonary vascular remodeling, and $\mathrm{PH}$, the pathological hallmarks of severe BPD (5-7).

Wnt/ $\beta$-catenin signaling is an early developmental pathway that plays an important role in airway and vascular smooth muscle cell development (8-10). Wnt signaling has been shown to be upregulated in the lungs of adult patients with idiopathic lung fibrosis and PH (11-13). Low-density lipoprotein receptor-related proteins 5 and 6 (LRP5/6) are Wnt coreceptors that bind to Wnt ligands and activate downstream Wnt signaling (14-17). LRP5/6 are critical mediators governing Wnt $/ \beta$-catenin signaling cascade in the vasculature and therefore play a critical role in vascular smooth muscle cell proliferation and survival $(18,19)$. We and others have shown that hyperoxia exposure in neonatal rats induces marked activation of Wnt/ $\beta$-catenin signaling as well as upregulation of LRP6 expression $(20,21)$. However, the role of LRP5/6 in the pathogenesis of BPD is unknown.

Mesd is a specialized chaperone for LRP5/6 that competitively blocks the binding of Wnt ligands to LRP5/6 and inhibits $\mathrm{Wnt} / \beta$-catenin signaling $(22,23)$. In the present study, we hypothesized that the inhibition of LRP5/6 by Mesd, a universal inhibitor of LRP 5/6, would prevent hyperoxia-induced Wnt/ $\beta$-catenin signaling and thereby protect against hyperoxia-induced lung injury in neonatal rats. Our results indicate that LRP5/6 mediate hyperoxia-induced pulmonary vascular remodeling and $\mathrm{PH}$, suggesting a potential therapeutic target to alleviate $\mathrm{PH}$ in neonates with severe $\mathrm{BPD}$.

\section{RESULTS}

Inhibition of LRP5/6 by Mesd Blocks Hyperoxia-Induced Activation of Wnt/ $\beta$-Catenin Signaling

First, we confirmed that inhibition of LRP5/6 by Mesd effectively blocks hyperoxia-induced activation of Wnt/ $\beta$-catenin 
a

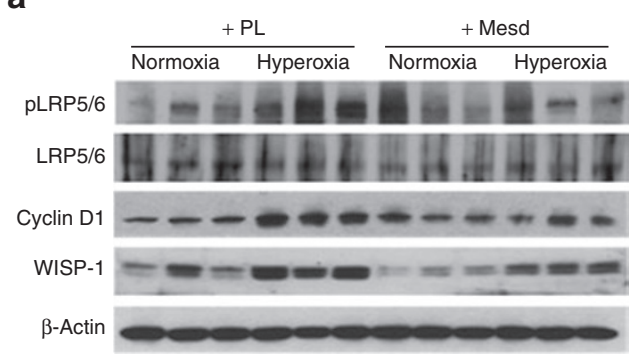

b

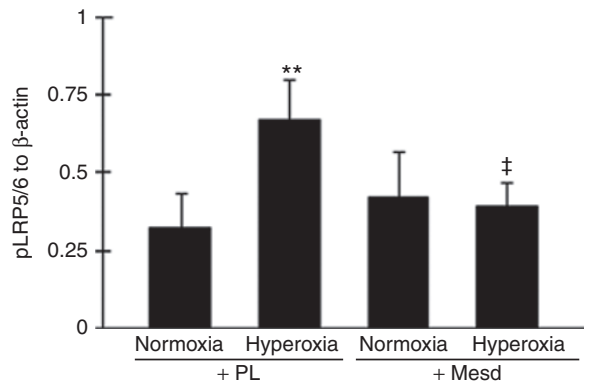

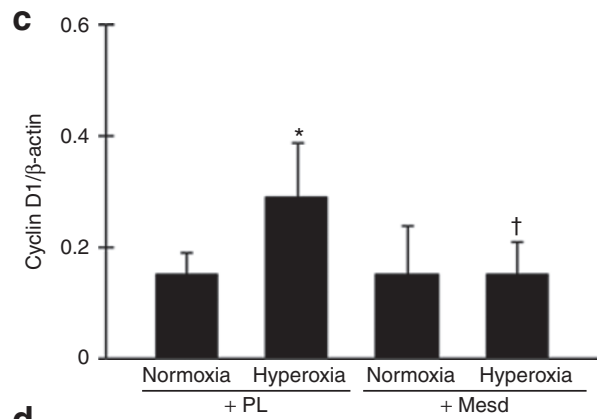

d

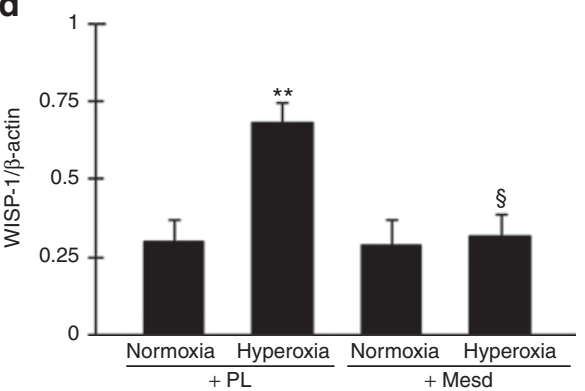

Figure 1. Mesd decreases pLRP5/6 and blocks expression of cyclin D1 and WISP-1. (a) Western blot analysis demonstrated significant increases of (b) pLRP5/6, (c) cyclin D1, and (d) WISP-1 expression in hyperoxia-exposed lungs as compared with normoxia lungs. Treatment with Mesd decreased expression of these genes during hyperoxia. Hyperoxia did not change the expression of LRP5/6. $n=6 /$ group. ${ }^{*} P<0.05$ and ${ }^{* *} P<0.001$ as compared with normoxia-exposed lungs; ${ }^{\dagger} P<0.05,{ }^{\ddagger} P<0.01$, and ${ }^{\S} P<0.001$ as compared with hyperoxia plus placebo lungs. $\mathrm{PL}$, placebo; pLRP5/6, phosphorylation of low-density lipoprotein receptor-related proteins 5 and 6; WISP, Wnt-induced signaling protein.

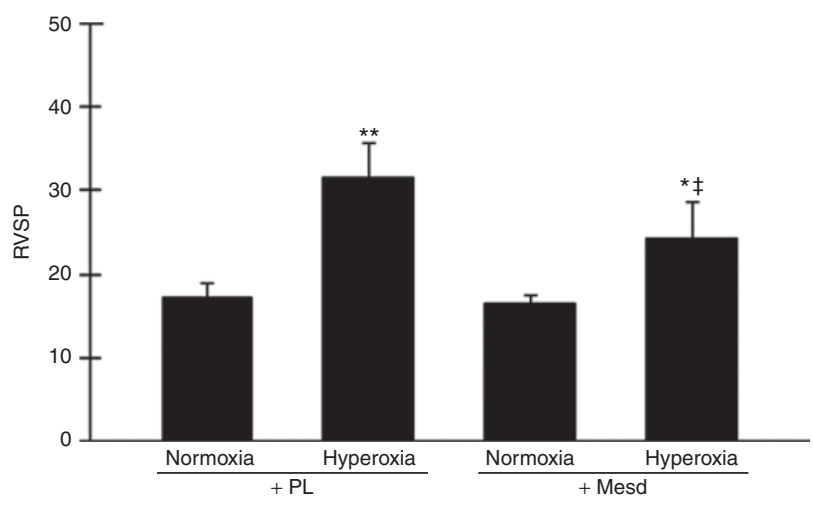

Figure 2. Mesd attenuates hyperoxia-induced PH. Hyperoxia exposure increased RVSP in the presence of placebo, which was attenuated by Mesd treatment. $n=5-6 /$ group. ${ }^{*} P<0.001$ and ${ }^{*} P<0.01$ as compared with normoxia lungs; ${ }^{\ddagger} P<0.01$ as compared with hyperoxia plus placebo lungs. $\mathrm{PH}$, pulmonary hypertension; $\mathrm{PL}$, placebo; RVSP, right-ventricular systolic pressure.

signaling by measuring LRP5/6 phosphorylation (pLRP5/6) and expression of $\mathrm{Wnt} / \beta$-catenin target genes, cyclin D1 and Wnt-induced signaling protein-1 (WISP-1). As shown in Figure 1b, hyperoxia exposure significantly increased pLRP5/6 without changing LRP5/6 expression in the lungs of animals treated with placebo, whereas administration of Mesd significantly decreased pLRP5/6. Correlating with LRP5/6 phosphorylation, hyperoxia also markedly increased cyclin D1 expression in the lungs of animals treated with placebo $(0.29 \pm 0.1 \mathrm{vs}$. $0.15 \pm 0.04, P<0.01$, hyperoxia plus placebo vs. normoxia plus placebo) (Figure 1c). However, administration of Mesd during hyperoxia suppressed cyclin D1 expression to normoxia levels $(0.15 \pm 0.06$ vs. $0.29 \pm 0.1, P<0.05$, hyperoxia plus Mesd vs. hyperoxia plus placebo). Similarly, expression of WISP-1, another immediate target gene of $\mathrm{Wnt} / \beta$-catenin signaling, was also markedly increased in hyperoxia placebo animals ( $0.68 \pm 0.07$ vs. $0.3 \pm 0.07, P<0.001$, hyperoxia plus placebo vs. normoxia plus placebo) and was suppressed to normoxia levels by treatment with Mesd $(0.32 \pm 0.07$ vs. $0.68 \pm 0.07, P<$ 0.001 , hyperoxia plus Mesd vs. hyperoxia plus placebo) (Figure 1d). Administration of Mesd did not affect pLRP5/6, cyclin D1, and WISP-1 expression during normoxia. Thus, inhibition of LRP5/ 6 by its universal inhibitor, Mesd, blocks hyperoxia activation of $\mathrm{Wnt} / \beta$-catenin signaling in neonatal rat lungs.

\section{LRP5/6 Inhibition Attenuates Hyperoxia-Induced PH}

Given the importance of Wnt/ $\beta$-catenin signaling in the development and progression of $\mathrm{PH}$ in adult patients as well as animal models, we sought to determine the effect of LRP5/6 inhibition on hyperoxia-induced $\mathrm{PH}$ by measuring right-ventricular systolic pressure (RVSP). RVSP was markedly increased in hyperoxia-exposed animals treated with placebo $(31.5 \pm 4.4$ vs. $17.2 \pm 1.8, P<0.001$, hyperoxia plus placebo vs. normoxia plus placebo) (Figure 2). By contrast, administration of Mesd decreased RVSP during hyperoxia $(24.3 \pm 4.5$ vs. $31.5 \pm 4.4, P<$ 0.01 , hyperoxia plus Mesd vs. hyperoxia plus placebo).

\section{LRP5/6 Inhibition Attenuates Hyperoxia-Induced Right- Ventricular Hypertrophy (RVH)}

Given that chronic $\mathrm{PH}$ induces right-ventricular remodeling and leads to $\mathrm{RVH}$, we next tested whether reduction of $\mathrm{PH}$ by LRP5/6 inhibition also attenuates RVH by measuring the weight ratio of the right ventricle to the left ventricle plus the 


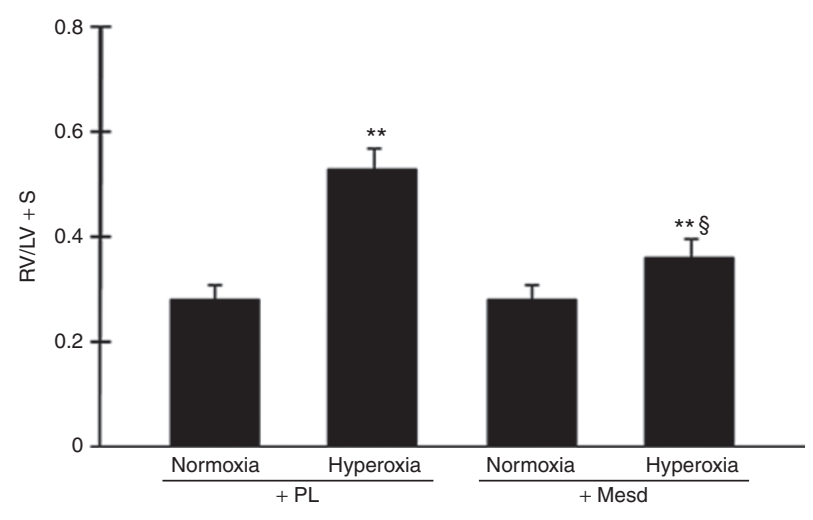

Figure 3. Mesd attenuates hyperoxia-induced RVH. Hyperoxia exposure increased RV/LV $+\mathrm{S}$ in the presence of placebo, which was attenuated by Mesd treatment. $n=6-8$ /group. ${ }^{* *} P<0.001$ as compared with normoxia lungs and ${ }^{5} P<0.001$ as compared with hyperoxia plus placebo lungs. $\mathrm{PL}$, placebo; RV/LV $+S$, ratio of right ventricle to left ventricle plus septum; $\mathrm{RVH}$, right-ventricular hypertrophy.

septum $(\mathrm{RV} / \mathrm{LV}+\mathrm{S})$. As hypothesized, although hyperoxia exposure induced marked RVH in placebo-treated animals $(0.53 \pm 0.04$ vs. $0.28 \pm 0.03, P<0.001$, hyperoxia plus placebo vs. normoxia plus placebo) (Figure 3), administration of Mesd significantly attenuated hyperoxia-induced RVH $(0.36 \pm 0.04$ vs. $0.53 \pm 0.04, P<0.001$, hyperoxia plus Mesd vs. hyperoxia plus placebo). Therefore, inhibition of LRP5/6 not only attenuates $\mathrm{PH}$ but also decreases RVH caused by hyperoxia.

\section{LRP5/6 Inhibition Decreases Pulmonary Vascular Remodeling}

$\mathrm{PH}$ associated with severe BPD is characterized by abnormal pulmonary vascular remodeling. To determine the role of LRP5/6 in pulmonary vascular remodeling, immunofluorescence staining for $\alpha$-smooth muscle actin ( $\alpha$-SMA) was performed on lung tissue sections, and medial wall thickness of peripheral pulmonary vessels of $<50 \mu \mathrm{m}$ in diameter was measured. Hyperoxia exposure significantly increased medial wall thickness in the presence of placebo $(0.22 \pm 0.04$ vs. $0.12 \pm 0.03, P<0.001$, hyperoxia plus placebo vs. normoxia plus placebo) (Figure 4), which was significantly attenuated when treated with the LRP5/6 inhibitor $(0.16 \pm 0.02$ vs. $0.22 \pm 0.04, P<0.05$, hyperoxia plus Mesd vs. hyperoxia plus placebo). These data indicate that LRP5/6 play a critical role in pulmonary vascular remodeling and may thus contribute to the development of $\mathrm{PH}$ during hyperoxia.

\section{LRP5/6 Inhibition Decreases $\beta$-Catenin Nuclear Translocation and} Proliferation of Pulmonary Vascular Smooth Muscle Cells

To address the underlying mechanisms by which LRP5/6 may mediate pulmonary vascular remodeling, we questioned whether LRP5/6 would regulate $\beta$-catenin nuclear translocation and proliferation of pulmonary vascular smooth muscle cells. As shown in Figure 5e, the percentage of peripheral pulmonary vessels with positive nuclear $\beta$-catenin was significantly increased by hyperoxia in the presence of placebo, whereas it was markedly decreased by treatment with Mesd. Double immunofluorescence staining with $a$-SMA and ki-67, a specific marker of cell proliferation, revealed a markedly increased
Normoxia $+\mathrm{PL}$

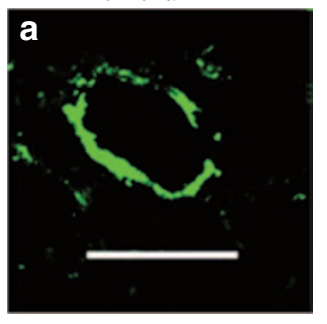

Normoxia + Mesd

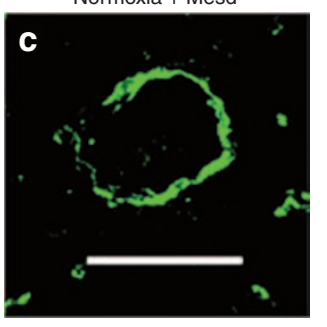

e

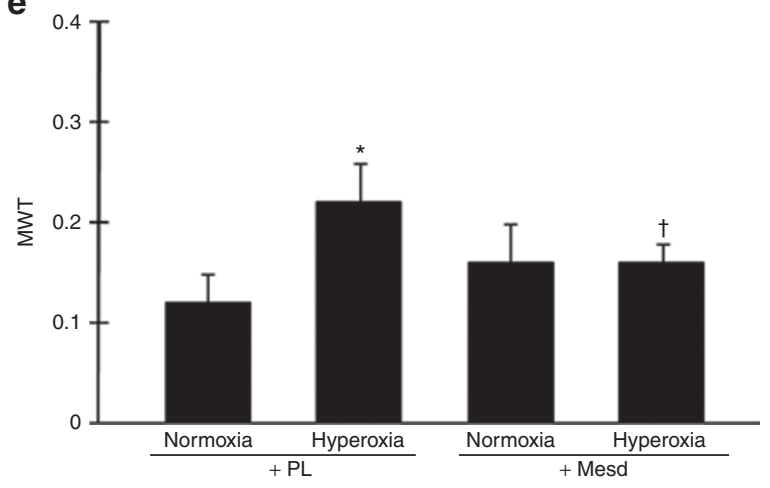

Figure 4. Mesd decreases pulmonary vascular remodeling. Immunostaining with an anti-a-SMA antibody (green signal) was performed to assess MWT of peripheral pulmonary vessels $(15-50 \mu \mathrm{m})$. (a) Normoxia + placebo; (b) hyperoxia + placebo; (c) normoxia + Mesd; and (d) hyperoxia + Mesd. (e) Hyperoxia significantly increased MWT in the presence of placebo, which was significantly decreased by Mesd administration. $n=4-6$ /group. ${ }^{*} P<0.05$ as compared with normoxia lungs and ${ }^{\dagger} P<0.05$ as compared with hyperoxia plus placebo lungs. Magnification: $\times 40$. Bar $=50 \mu \mathrm{m}$. MWT, medial wall thickness; $\mathrm{PL}$, placebo; SMA, smooth muscle actin.

percentage of proliferating $\alpha$-SMA positive cells in the pulmonary vascular medial wall in hyperoxia-exposed and placebotreated animals (Figure 5j), whereas administration of Mesd decreased the percentage of proliferating a-SMA positive cells in the pulmonary vascular medial wall during hyperoxia exposure $(0.13 \pm 0.05$ vs. $0.21 \pm 0.04, P<0.01$, hyperoxia plus Mesd vs. hyperoxia plus placebo). This suggests that LRP5/6 regulate proliferation of pulmonary vascular smooth muscle cells that may be at least partially responsible for the increased muscularization and remodeling of peripheral pulmonary vessels.

\section{Effect of LRP5/6 Inhibition on Vascularization and Alveolar} Development

Decreased vascular growth also contributes to the development of PH in severe BPD. To assess the effect of LRP5/6 inhibition on vascular development, we performed immunofluorescence staining with an anti-von Willebrand factor antibody. 


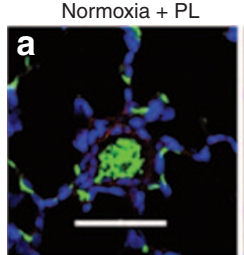

Normoxia + Mesd

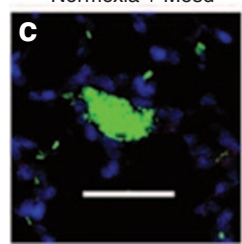

Normoxia + PL
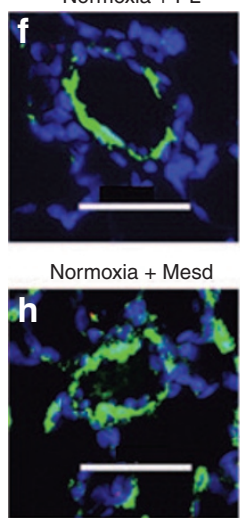

Hyperoxia + PL

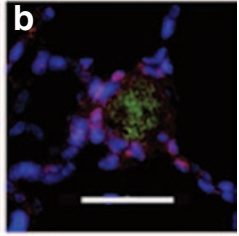

Hyperoxia + Mesd

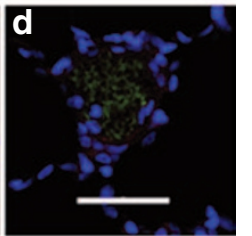

Hyperoxia + PL

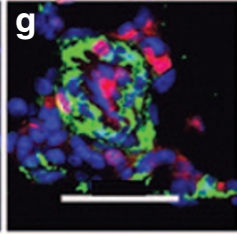

Hyperoxia + Mesd

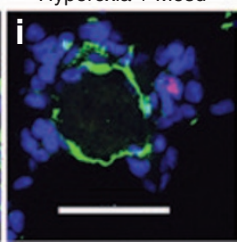

e

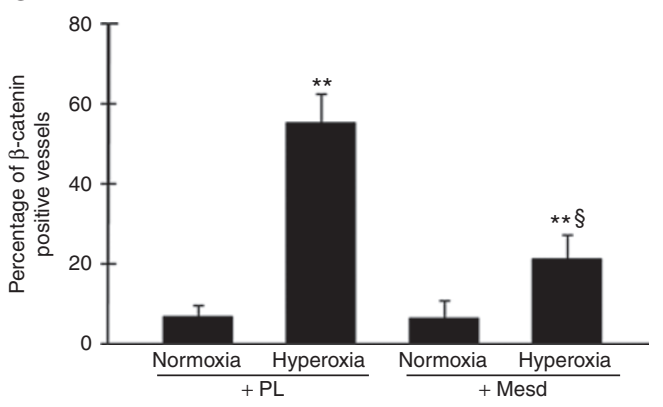

j

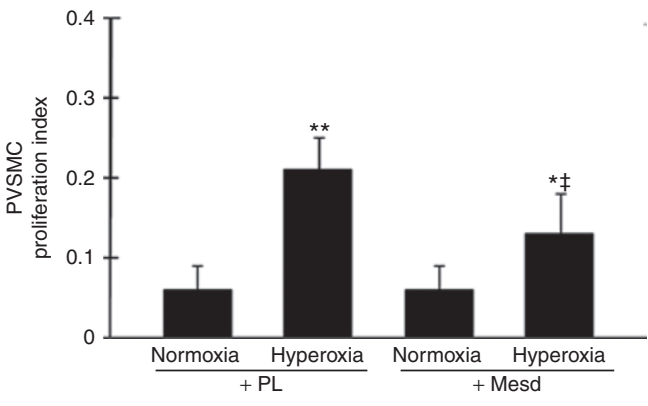

Figure 5. Mesd decreases $\beta$-catenin nuclear translocation and proliferation of PVSMC. (a) Immunofluorescence staining for $\beta$-catenin (red signaling) and DAPI nuclear staining (blue signal) in lungs from normoxia plus placebo, (b) hyperoxia plus placebo, (c) normoxia plus Mesd, and (d) hyperoxia plus

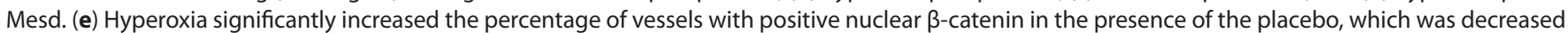
by treatment with Mesd. Double immunofluorescence staining for a-SMA (green signal) and ki-67 (red signal) and DAPI nuclear staining (blue signal) in lungs from (f) normoxia plus placebo, (g) hyperoxia plus placebo, (h) normoxia plus Mesd, and (i) hyperoxia plus Mesd. (j) The index of ki-67 positive nuclei (pink signal) in PVSMC was significantly increased in hyperoxia plus placebo lungs, which was decreased by administration of Mesd. $n=5 / g$ roup. ${ }^{*} P<0.001$ and ${ }^{*} P<0.05$ as compared with normoxia lungs; ${ }^{\S} P<0.001$ and ${ }^{\ddagger} P<0.01$ as compared with hyperoxia plus placebo lungs. Bar $=50 \mu \mathrm{m}$. $\mathrm{PL}$, placebo; PVSMC, pulmonary vascular smooth muscle cells; SMA, smooth muscle actin.

Compared with normoxia lungs, the number of von Willebrand factor-positive vessels was significantly decreased in hyperoxia lungs exposed to placebo $(2.8 \pm 0.7$ vs. $8.9 \pm 0.8, P<0.001$, hyperoxia plus placebo vs. normoxia plus placebo). However, no significant change was noted after Mesd treatment during hyperoxia ( $2.5 \pm 0.7$ vs. $2.8 \pm 0.7, P=0.5$, hyperoxia plus Mesd vs. hyperoxia plus placebo). Similarly, LRP5/6 inhibition did not improve alveolarization. Although mean linear intercept was increased by hyperoxia exposure in placebo-treated animals $(59.7 \pm 3.9$ vs. $42 \pm 2.2, P<0.001$, hyperoxia plus placebo vs. normoxia plus placebo), no effect was noted after administration of Mesd (60.8 \pm 3.7 vs. 59 . $\pm 3.9, P=0.5$, hyperoxia plus Mesd vs. hyperoxia plus placebo). As shown in other studies, expression of vascular endothelial growth factor (VEGF) was markedly decreased in hyperoxia placebo lungs. However, administration of Mesd did not change VEGF expression during hyperoxia (data not shown).

\section{DISCUSSION}

The present study demonstrates that LRP5/6 inhibition by Mesd blocks hyperoxia-induced Wnt/ $\beta$-catenin signaling in neonatal rat lungs and attenuates chronic hyperoxia-induced $\mathrm{PH}, \mathrm{RVH}$, pulmonary vascular remodeling, and pulmonary vascular smooth muscle cell proliferation. However, LRP5/6 inhibition by Mesd did not improve alveolarization or vascularization. Collectively, these data indicate a critical role of LRP5/6 in the pathogenesis of pulmonary vascular remodeling and $\mathrm{PH}$ as a result of chronic hyperoxia exposure, thereby suggesting a potential therapeutic target to alleviate $\mathrm{PH}$ in neonates with severe BPD.

$\mathrm{PH}$ contributes significantly to the mortality and morbidity of preterm infants with severe BPD despite advances in neonatal intensive care and widespread availability and use of pulmonary vasodilators such as nitric oxide $(4,24,25)$. Clinical efforts to prevent and treat BPD and PH have been unsuccessful because of its multifactorial nature and poorly understood pathogenic mechanisms. Hyperoxia-induced lung injury in neonatal rats has been widely used as an experimental model of BPD to explore disease processes and identify potential therapeutic targets.

The Wnt/ $\beta$-catenin signaling pathway is a developmentally active pathway that has been linked to the pathogenesis of 
important lung diseases such as pulmonary fibrosis and $\mathrm{PH}$ (1113). LRP5/6 are Wnt coreceptors that bind to Wnt ligands and activate $\beta$-catenin nuclear translocation and further signaling (14-17). Although Wnt/ $\beta$-catenin signaling proteins are present in detectable levels under normal conditions, their expression is markedly increased in disease states. Cyclin D1 and WISP-1 are two important target genes of the Wnt $/ \beta$-catenin signaling that regulate a diverse array of cell function and differentiation. Specifically, both cyclin D1 and WISP-1 have been implicated as playing critical roles in pulmonary vascular smooth muscle cell proliferation and differentiation $(26,27)$.

Recently, we and others have shown that in a hyperoxiainduced rat model of BPD, expression of LRP6 and Wnt/ $\beta$ catenin signaling proteins is markedly increased $(20,21)$. Mesd is a gene identified in the mesoderm development deletion interval on mouse chromosome 7. Mesd functions as specific chaperone for LRP5/6 and is essential for specification of embryonic polarity and mesoderm induction $(22,23,28,29)$. To better elucidate the role of LRP5/6 in neonatal hyperoxiainduced lung injury, we tested whether inhibition of Wnt $/ \beta$ catenin signaling by blocking LRP5/6 receptors with Mesd would prevent hyperoxia-induced lung injury. We have shown for the first time that in a neonatal hyperoxia-induced lung injury model, treatment with Mesd effectively decreases LRP5/6 phosphorylation and inhibits the expression of Wnt/ $\beta$ catenin target genes, cyclin D1, and WISP-1.

The primary finding of this study is that inhibition of LRP5/6mediated $W n t / \beta$-catenin signaling by Mesd attenuated hyperoxia-induced $\mathrm{PH}$. Both excessive pulmonary vascular remodeling and impaired vascular development are associated with $\mathrm{PH}$ in BPD. PH may be caused by decreased pulmonary vasculature that limits vascular surface area, thus leading to elevation of pulmonary vascular resistance (4). The excessive pulmonary vascular remodeling may further contribute to high pulmonary vascular resistance through narrowing of the vessel diameter and decreased vascular compliance (4). This study demonstrates that LRP5/6 inhibition attenuates hyperoxia-induced $\mathrm{PH}$ and $\mathrm{RVH}$ primarily by inhibiting pulmonary vascular remodeling. The medial wall thickness and smooth muscle cell proliferation of peripheral pulmonary vessels were significantly decreased by Mesd treatment during hyperoxia. This finding is in agreement with recent studies that have suggested an important role of LRP5/6 and Wnt/ $\beta$-catenin signaling in vascular smooth muscle cell development and $\mathrm{PH}$. Gene expression analysis of pulmonary arterial resistance vessels revealed differentially regulated canonical and noncanonical Wht genes in $\mathrm{PH}(13,19,27,30,31)$.

A distinct feature of $\mathrm{PH}$ is the increased abundance of $\alpha$-SMA-positive cells in the vessel wall. In the current study, we noted a significant increase in the percentage of proliferating a-SMA-positive cells in the pulmonary vascular medial wall on exposure to hyperoxia. These a-SMA-positive cells may originate from the proliferation of resident vascular smooth muscle cells, adventitial fibroblasts, recruitment of vascular progenitors cells, and transition of endothelial cells into a mesenchymal phenotype (32-35). Inhibition of LRP5/6 during hyperoxia resulted in a decrease in the percentage of proliferating a-SMA cells in the pulmonary vascular medial wall. Furthermore, this decreased proliferation is correlated with decreased $\beta$-catenin nuclear translocation in the vascular walls. These results highlight the role of LRP5/6 in regulating the proliferation and survival of resident vascular smooth muscle cells (19). However, whether LRP5/6 play a role in the recruitment of vascular progenitor cells and endothelial-tomesenchymal transition is not clear. It is possible that the partial effect of LRP inhibition on proliferation of pulmonary vascular a-SMA-positive cells as observed in our study is caused by its specific effect on resident pulmonary vascular smooth muscle cells. Mechanisms involved in vascular progenitor cell recruitment and endothelial-to-mesenchymal transition may be independent of LRP5/6 and require further investigation.

We did not observe any beneficial effect of LRP5/6 inhibition on hyperoxia-induced impairment of pulmonary vascularization. The $\mathrm{Wnt} / \beta$-catenin pathway is thought to play a critical role in angiogenesis by regulating VEGF, particularly in retinal development (36). VEGF is the most important factor in pulmonary vascular development $(37,38)$. Although VEGF expression was decreased by hyperoxia in this study, treatment with Mesd did not augment VEGF expression during hyperoxia. This suggests that the mechanisms involved in hyperoxia-induced downregulation of VEGF and impairment of vascularization may occur through pathways independent of LRP5/6-modulated Wnt/ $\beta$-catenin signaling and may also explain the partial effect of Mesd administration on $\mathrm{PH}$.

Impaired alveolarization is one of the pathological hallmarks of clinical as well as experimental models of BPD $(1,7)$. The importance of Wnt/ $\beta$-catenin signaling in alveolar development has been demonstrated by previous genetic loss-of-function and gain-of-function studies $(10,39)$. Dasgupta et al. have also reported that the administration of peroxisome proliferatoractivated receptor- $\gamma$ agonist, rosiglitazone, decreases hyperoxia activation of Wnt $/ \beta$-catenin and transforming growth factor- $\beta$ signaling and improves alveolar development (21). Our study did not show improvement of alveolarization by treatment with Mesd during hyperoxia. Our results clearly demonstrate the profound effect on vascular remodeling and the lack of effect on alveolar as well as vascular development by inhibition of LRP5/6 during hyperoxia. One possible explanation is that LRP5/6 inhibition is specific for canonical Wnt $/ \beta$-catenin signaling. The other possibility is the lack of effect of LRP5/6 inhibition on VEGF expression because VEGF is not only important for angiogenesis but also for alveolarization. There could also be other unknown mechanisms, such as the difference in the abundance of LRP5/6 expression among different types of lung cells.

In conclusion, this study demonstrates that LRP5/6 mediates pulmonary vascular remodeling and $\mathrm{PH}$ in hyperoxia-induced neonatal lung injury and that inhibition of this pathway attenuates $\mathrm{PH}$. In addition, this study also shows that inhibition of LRP5/6-mediated Wnt/ $\beta$-catenin signaling does not have a deleterious effect on normal alveolar and vascular development. Our data suggest that therapeutic interventions targeting LRP5/6 may be a beneficial adjunct in the treatment of neonatal PH associated with BPD because currently available 
therapies consist primarily of vasodilators that have minimal effect on reducing pulmonary vascular remodeling.

\section{METHODS}

\section{Animal Model and Experimental Protocol}

Timed pregnant Sprague-Dawley rats (The Jackson Laboratory, Bar Harbor, ME) were delivered naturally at term gestation. Newborn rat pups were randomized within $24 \mathrm{~h}$ after birth to four groups to receive normoxia $\left(21 \% \mathrm{O}_{2}\right)$ plus phosphate-buffered saline (PBS) (placebo); normoxia plus Mesd; hyperoxia $\left(90 \% \mathrm{O}_{2}\right)$ plus PBS; or hyperoxia plus Mesd. Recombinant mouse Mesd protein was prepared as described previously (23). Mesd (10 mg/kg mixed in PBS) or PBS (equal volume) was given by intraperitoneal injection before exposure to hyperoxia and then every other day for a total of $14 \mathrm{~d}$. Hyperoxia exposure was achieved in a sealed plastic chamber with continuous $\mathrm{O}_{2}$ monitoring. The study protocol was approved by the University of Miami Animal Care and Use Committee, and all animals were cared for according to the guidelines set forth by the institution and the US National Institutes of Health.

\section{Hemodynamic Measurements}

On postnatal day 15 , animals were anesthetized and RVSP was measured by cardiac catheterization as previously described (40). Hearts were then dissected and the RV/LV $+\mathrm{S}$ weight ratio was determined as an index for RVH (40).

\section{Lung Tissue Collection}

For histological and morphometric analyses, lungs were infused with $4 \%$ paraformaldehyde in PBS via a tracheal catheter under $20 \mathrm{~cm} \mathrm{H}_{2} \mathrm{O}$ pressure for $5 \mathrm{~min}$ and then fixed in $4 \%$ paraformaldehyde overnight at $4{ }^{\circ} \mathrm{C}$. Dehydrated lung tissues were paraffin embedded and 5 - $\mu \mathrm{m}$ tissue sections were prepared.

\section{Lung Morphometry}

Hematoxylin and eosin-stained tissue sections were used to measure the mean linear intercept (40).

\section{Double Immunofluorescence Staining}

Double immunofluorescence staining was performed as previously described (40). The following primary antibodies were used: mouse anti- $\beta$-catenin and anti- $\alpha$-SMA antibodies from Sigma (Saint Louis, MO); a rabbit anti-von Willebrand factor antibody from Dako (Carpenteria, CA); and a rabbit anti-ki-67 antibody from Abcam (Cambridge, MA).

\section{Assessment of Pulmonary Vascular Remodeling, $\beta$-Catenin Nuclear Translocation, and Proliferation \\ Medial wall thickness of peripheral pulmonary vessels $(<50 \mu \mathrm{m})$ was assessed on $\alpha$-SMA-stained lung tissue sections (40). The percentage of peripheral vessels with at least one $\beta$-catenin positive nucleus was determined on lung tissue sections with $\beta$-catenin immunofluores- cence and DAPI nuclear staining. Proliferation of pulmonary vascular smooth muscle cells was determined on a-SMA and ki-67 (marker for cell proliferation) double-stained lung tissue sections. The pro- liferation index was determined by the ratio of smooth muscle cells with ki-67 positive nucleus to total smooth muscle cells on 20 vessels $(<50 \mu \mathrm{m})$ per slide.}

\section{Assessment of Vascular Density}

Vascular density was assessed on von Willebrand factor-stained lung sections as previously described (40).

\section{Western Blot Analysis}

Total protein extraction and western blot analysis were performed as previously described (40). Primary antibodies used included cyclin D1, WISP-1, and VEGF from Santa Cruz Biotechnology (Santa Cruz, CA) and pLRP5/6 from Cell Signaling (Danvers, MA).

\section{Statistical Analysis}

Data are expressed as means $\pm \mathrm{SD}$. Comparison among groups was performed by one-way ANOVA followed by the Student-NewmanKeuls test. $P<0.05$ was considered significant.

\section{STATEMENT OF FINANCIAL SUPPORT}

This study was supported by an Advancing Newborn Medicine Fellow Research Award, IKARIA (to D.A.), a Grant-in-Aid from the American Heart Association (to S.W.), a Batchelor Award (to S.W.), and Project Newborn from the University of Miami (to S.W.).

Disclosure: Y.L. is one of two inventors on a patent for the use of Mesd peptide in bone disease.

\section{REFERENCES}

1. Jobe AH, Bancalari E. Bronchopulmonary dysplasia. Am J Respir Crit Care Med 2001;163:1723-9.

2. Van Marter LJ. Epidemiology of bronchopulmonary dysplasia. Semin Fetal Neonatal Med 2009;14:358-66.

3. Bhandari A, Bhandari V. Pitfalls, problems, and progress in bronchopulmonary dysplasia. Pediatrics 2009;123:1562-73.

4. Stenmark KR, Abman SH. Lung vascular development: implications for the pathogenesis of bronchopulmonary dysplasia. Annu Rev Physiol 2005; 67:623-61.

5. Warner BB, Stuart LA, Papes RA, Wispé JR. Functional and pathological effects of prolonged hyperoxia in neonatal mice. Am J Physiol 1998;275(1 Pt 1):L110-7.

6. Horowitz S. Pathways to cell death in hyperoxia. Chest 1999;116:Suppl 1:64S-7S.

7. Bhandari V. Hyperoxia-derived lung damage in preterm infants. Semin Fetal Neonatal Med 2010;15:223-9.

8. Morrisey EE. Wnt signaling and pulmonary fibrosis. Am J Pathol 2003;162:1393-7.

9. Van Scoyk M, Randall J, Sergew A, Williams LM, Tennis M, Winn RA. Wnt signaling pathway and lung disease. Transl Res 2008;151:175-80.

10. Königshoff M, Eickelberg O. WNT signaling in lung disease: a failure or a regeneration signal? Am J Respir Cell Mol Biol 2010;42:21-31.

11. Chilosi M, Poletti V, Zamò A, et al. Aberrant Wnt/beta-catenin pathway activation in idiopathic pulmonary fibrosis. Am J Pathol 2003;162: 1495-502.

12. Königshoff M, Balsara N, Pfaff EM, et al. Functional Wnt signaling is increased in idiopathic pulmonary fibrosis. PLoS ONE 2008;3:e2142.

13. Cohen ED, Ihida-Stansbury K, Lu MM, Panettieri RA, Jones PL, Morrisey EE. Wnt signaling regulates smooth muscle precursor development in the mouse lung via a tenascin C/PDGFR pathway. J Clin Invest 2009; 119:2538-49.

14. Tamai K, Semenov M, Kato Y, et al. LDL-receptor-related proteins in Wnt signal transduction. Nature 2000;407:530-5.

15. Pinson KI, Brennan J, Monkley S, Avery BJ, Skarnes WC. An LDL-receptorrelated protein mediates Wnt signalling in mice. Nature 2000;407:535-8.

16. Mao B, Wu W, Li Y, et al. LDL-receptor-related protein 6 is a receptor for Dickkopf proteins. Nature 2001;411:321-5.

17. Mao J, Wang J, Liu B, et al. Low-density lipoprotein receptor-related protein- 5 binds to Axin and regulates the canonical Wnt signaling pathway. Mol Cell 2001;7:801-9.

18. He X, Semenov M, Tamai K, Zeng X. LDL receptor-related proteins 5 and 6 in Wnt/beta-catenin signaling: arrows point the way. Development 2004;131:1663-77.

19. Wang X, Adhikari N, Li Q, Hall JL. LDL receptor-related protein LRP6 regulates proliferation and survival through the Wnt cascade in vascular smooth muscle cells. Am J Physiol Heart Circ Physiol 2004;287: H2376-83.

20. Alapati D, Rong M, Chen S, et al. Connective tissue growth factor antibody therapy attenuates hyperoxia-induced lung injury in neonatal rats. Am J Respir Cell Mol Biol 2011;45:1169-77.

21. Dasgupta C, Sakurai R, Wang Y, et al. Hyperoxia-induced neonatal rat lung injury involves activation of TGF- $\{$ beta $\}$ and Wnt signaling and is protected by rosiglitazone. Am J Physiol Lung Cell Mol Physiol 2009;296:L1031-41. 
22. Hsieh JC, Lee L, Zhang L, et al. Mesd encodes an LRP5/6 chaperone essential for specification of mouse embryonic polarity. Cell 2003;112:355-67.

23. Lu W, Liu CC, Thottassery JV, Bu G, Li Y. Mesd is a universal inhibitor of Wnt coreceptors LRP5 and LRP6 and blocks Wnt/beta-catenin signaling in cancer cells. Biochemistry 2010;49:4635-43.

24. Fouron JC, Le Guennec JC, Villemant D, Perreault G, Davignon A. Value of echocardiography in assessing the outcome of bronchopulmonary dysplasia of the newborn. Pediatrics 1980;65:529-35.

25. Thébaud B, Abman SH. Bronchopulmonary dysplasia: where have all the vessels gone? Roles of angiogenic growth factors in chronic lung disease. Am J Respir Crit Care Med 2007;175:978-85.

26. Königshoff M, Kramer M, Balsara N, et al. WNT1-inducible signaling protein-1 mediates pulmonary fibrosis in mice and is upregulated in humans with idiopathic pulmonary fibrosis. J Clin Invest 2009;119:772-87.

27. Quasnichka H, Slater SC, Beeching CA, Boehm M, Sala-Newby GB, George SJ. Regulation of smooth muscle cell proliferation by beta-catenin/ T-cell factor signaling involves modulation of cyclin D1 and p21 expression. Circ Res 2006;99:1329-37.

28. Lin $\mathrm{C}, \mathrm{Lu} \mathrm{W}$, Zhai L, et al. Mesd is a general inhibitor of different Wnt ligands in Wnt/LRP signaling and inhibits PC-3 tumor growth in vivo. FEBS Lett 2011;585:3120-5.

29. Li Y, Lu W, He X, Bu G. Modulation of LRP6-mediated Wnt signaling by molecular chaperone Mesd. FEBS Lett 2006;580:5423-8.

30. Wang Z, Shu W, Lu MM, Morrisey EE. Wnt7b activates canonical signaling in epithelial and vascular smooth muscle cells through interactions with Fzd1, Fzd10, and LRP5. Mol Cell Biol 2005;25:5022-30.

31. Laumanns IP, Fink L, Wilhelm J, et al. The noncanonical WNT pathway is operative in idiopathic pulmonary arterial hypertension. Am J Respir Cell Mol Biol 2009;40:683-91.
32. Haworth SG. Pulmonary vascular remodeling in neonatal pulmonary hypertension. State of the art. Chest 1988;93:Suppl 3:133S-8S.

33. Humbert M, Morrell NW, Archer SL, et al. Cellular and molecular pathobiology of pulmonary arterial hypertension. J Am Coll Cardiol 2004;43:Suppl $12 \mathrm{~S}: 13 \mathrm{~S}-24 \mathrm{~S}$.

34. Stenmark KR, Fagan KA, Frid MG. Hypoxia-induced pulmonary vascular remodeling: cellular and molecular mechanisms. Circ Res 2006; 99:675-91.

35. Sakao S, Tatsumi K, Voelkel NF. Reversible or irreversible remodeling in pulmonary arterial hypertension. Am J Respir Cell Mol Biol 2010;43: 629-34.

36. Dejana E. The role of wnt signaling in physiological and pathological angiogenesis. Circ Res 2010;107:943-52.

37. Le Cras TD, Markham NE, Tuder RM, Voelkel NF, Abman SH. Treatment of newborn rats with a VEGF receptor inhibitor causes pulmonary hypertension and abnormal lung structure. Am J Physiol Lung Cell Mol Physiol 2002;283:L555-62.

38. Bhatt AJ, Pryhuber GS, Huyck H, Watkins RH, Metlay LA, Maniscalco WM. Disrupted pulmonary vasculature and decreased vascular endothelial growth factor, Flt-1, and TIE-2 in human infants dying with bronchopulmonary dysplasia. Am J Respir Crit Care Med 2001;164(10 Pt 1): 1971-80.

39. Mucenski ML, Nation JM, Thitoff AR, et al. Beta-catenin regulates differentiation of respiratory epithelial cells in vivo. Am J Physiol Lung Cell Mol Physiol 2005;289:L971-9.

40. Chen S, Rong M, Platteau A, et al. CTGF disrupts alveolarization and induces pulmonary hypertension in neonatal mice: implication in the pathogenesis of severe bronchopulmonary dysplasia. Am J Physiol Lung Cell Mol Physiol 2011;300:L330-40. 